This item was submitted to Loughborough's Research Repository by the author.

Items in Figshare are protected by copyright, with all rights reserved, unless otherwise indicated.

\title{
Implications for SAR when using a symmetric phantom exposed to RF radiation using the FDTD method
}

PLEASE CITE THE PUBLISHED VERSION

LICENCE

CC BY-NC-ND 4.0

\section{REPOSITORY RECORD}

Whittow, W.G., and R.M. Edwards. 2019. "Implications for SAR When Using a Symmetric Phantom Exposed to RF Radiation Using the FDTD Method". figshare. https://hdl.handle.net/2134/2898. 
This item was submitted to Loughborough's Institutional Repository by the author and is made available under the following Creative Commons Licence conditions.

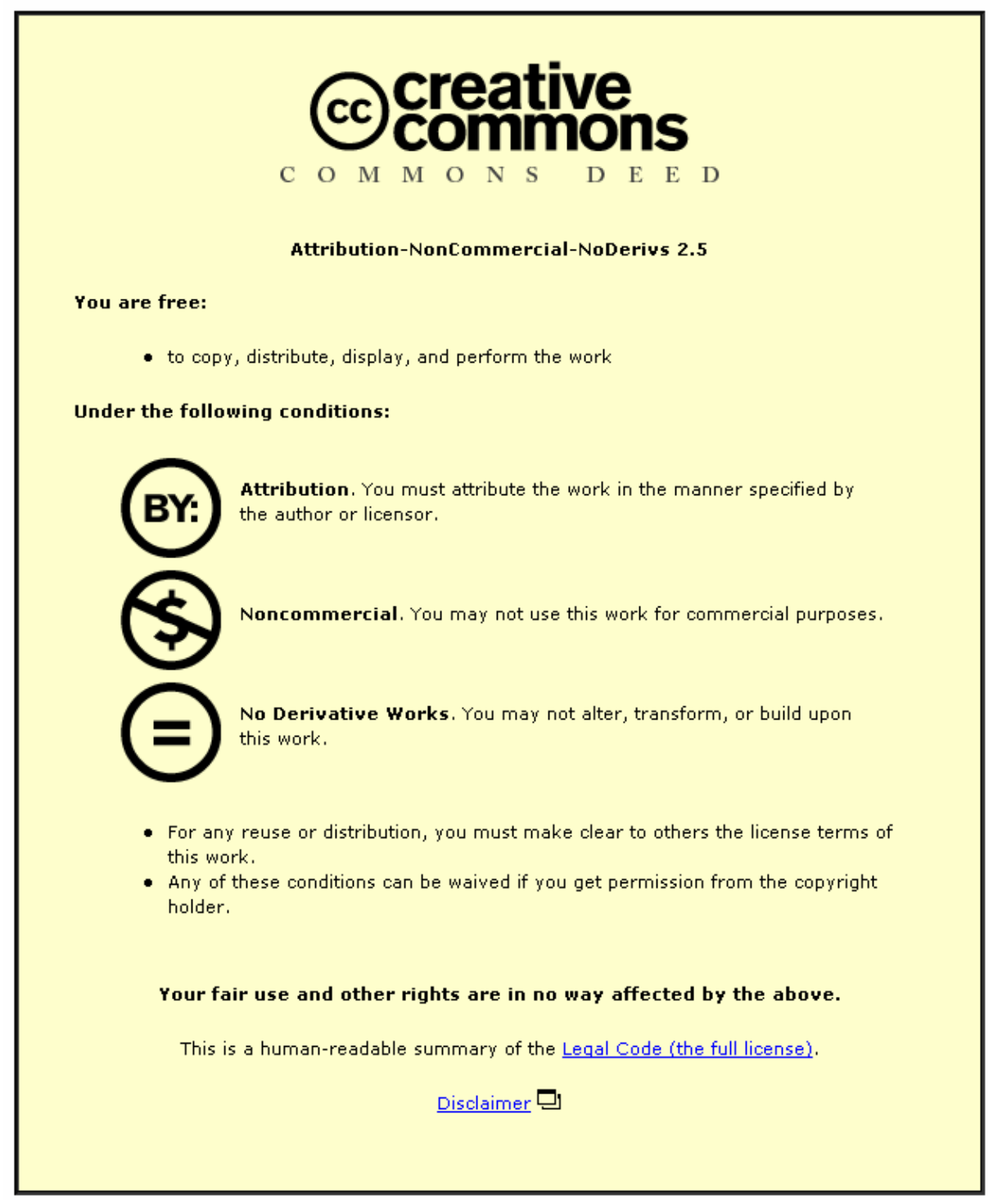

For the full text of this licence, please go to: http://creativecommons.org/licenses/by-nc-nd/2.5/ 


\title{
IMPLICATIONS FOR SAR WHEN USING A SYMMETRIC PHANTOM EXPOSED TO RF RADIATION USING THE FDTD METHOD
}

\author{
W. G. Whittow and R. M. Edwards
}

University of Sheffield, UK

Abstract - this paper investigates the implications of SAR when using a symmetric head compared to a whole head. The excitation is a vertically polarised plane wave traveling from the front of the face to the back of the head. The frequency range considered is 1.5 to $3.0 \mathrm{GHz}$. In general actual human heads are not symmetric. For example one eye may be slightly higher than the other and a nose maybe somewhat bent. Recently researchers also have evidence of brain torque (i.e. rightward frontal and leftward occipital asymmetry) in humans. In this FDTD model the head is modeled as two identical mirrored halves. By this method the field values need not be calculated twice thereby reducing computation time and memory requirements. Our results show whole head versus mirrored head comparisons for the maximum 1 and $10 \mathrm{~g}$ SAR in the head and gives particular attention to the SAR in the eyes.

Index Terms-FDTD, SAR, eye, symmetry

\section{INTRODUCTION}

The study of interactions between biological material and the energy generated by personal communications devices is currently topical. This paper investigates the effects of symmetry with a CW source positioned in front of the head. A rigorous Finite-Difference Time-Domain (FDTD) model is used. In recent years some work has been written up regarding mobile phones positioned near the ear [1-4]. The head has also been irradiated from in front of the eye using realistic mobile phone models [3-5]. This is topical as such hand held devices held to the front of and away from the head may soon become popular. Dimbylow and Hirata have illuminated the head with a plane wave from the front $[6,7]$ and have previously found resonance in the eyes within typical cellular spectra. Bernardi [8] considered the eyes to be particularly sensitive organs due to their proximity to the surface of the head and the their relatively low levels of blood flow when compared to other regions of the body. Dimbylow [3] also stresses the vulnerability of the eyes as they have a tendency to accumulate damage and cellular debris. EM modeling is computationally expensive; it is therefore advantageous to use symmetry which can reduce computational costs.

\section{DESCRIPTION OF MODEL}

An independent 3D FDTD code has been written; see Taflove [9] for an excellent reference. Perfectly Matched Layers (PML), with geometric grading [10], absorbing boundary conditions are used to terminate the grid. The PML is eight cells thick and is positioned ten cells from the head. The FDTD grid size was $174 \times 140 \times 140$ cells in the $x, y$, and $z$ dimensions.

A plane wave is injected into the grid using the total field / scattered field approach [9]. This produces a $z$ polarised plane wave propagating in the $x$ direction (from the nose to the rear of the head). See Fig. 1 for orientation of the axes. The power density used was $50 \mathrm{~W} / \mathrm{m}^{2}$, the maximum permissible exposure limit in controlled environments [7].

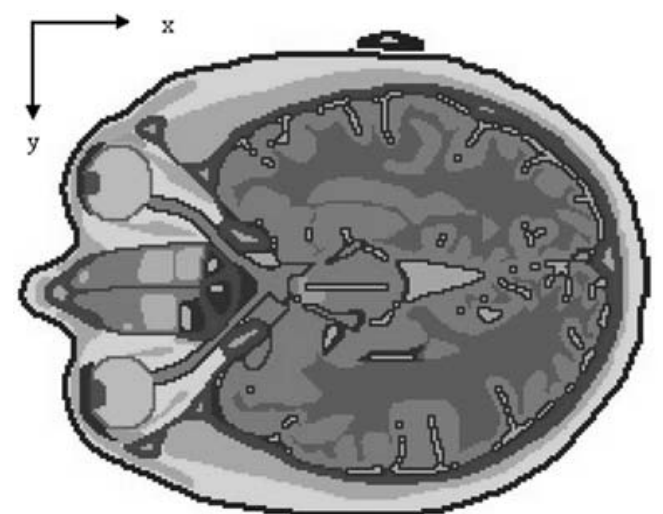

Fig. 1. A cross section through the eyes of the Brooks head of $2 \mathrm{~mm}$ resolution.

A head matrix provided by Brooks Air force [11] was used. The head, which is based on MRI data, is that of an adult male and has twenty-five tissue types. Fig. 1 shows a cross section of the $2 \mathrm{~mm}$ head through the centre of the eyes. The layer of fat, muscle and skin in front of the eye can be seen which make up the eyelids. It should be noted that the Brooks head has closed eyelids and the results in this paper are calculated with the eyelids kept closed. Hirata showed [3] that opening the eyelid increased the average SAR in the eye by $15 \%$ at $5.0 \mathrm{GHz}$.

The size of the Yee cell was $2 \mathrm{~mm}$, equal to the resolution of the head. The lowest number of cells per wavelength was always greater than six, and reasonable results have been obtained with only four [6]. 
Fig. 1 shows that the Brooks head is not exactly symmetric; for example there are visible differences in the eyes, sinuses and the top of one ear is higher than the other. A line of symmetry, in the $x-z$ plane, has been included in this model to save memory and computational time. This plane of symmetry runs vertically down the centre of the nose and extends to the back of the head. This was achieved by replacing the mid-plane of the grid with a magnetic wall $[7,12]$, and thus assuming the other half of the head is identical.

The dielectric properties are calculated with aid of the 4Cole-Cole extrapolation [13] and are frequency dependant. At the interface between two materials, the average values of conductivity and permittivity are used. The densities of the different materials are the same as used by Mason [14].

SAR is the standard criteria to measure the amount of electromagnetic energy absorbed in the body and is calculated as in equation (1)

$$
S A R=\frac{\sigma|E|^{2}}{\rho} \quad(\mathrm{W} / \mathrm{Kg})
$$

Where $|E|^{2}$ is the root mean square of the electric field components, $\rho$ is the density of the material in $\mathrm{Kg} / \mathrm{m}^{3}$ and $\sigma$ is the conductivity in $\mathrm{S} / \mathrm{m}$. The SAR is calculated with the twelve-field approach as used by Caputa [15].

In previous works [16] the FDTD, code including the PML absorbing boundary conditions, has been validated. The average SAR in an anatomical head excited with $E z$ and Ey polarized plane waves as a function of frequency showed good agreement with Hirata [7].

\section{RESULTS}

To examine the effects of symmetry, four metrics were investigated; the maximum local SAR in a single cell, the average SAR in the eye, the SAR ${ }_{1 G}$ averaged over one gram and the $S A R_{10 \mathrm{G}}$ averaged over ten grams. The SAR $\mathrm{R}_{1 \mathrm{G}}$ and SAR ${ }_{10 \mathrm{G}}$ have been averaged over a cubic volume containing no air [15] and have been calculating assuming the head is symmetric.

\section{A. Average SAR in the Eye}

The eye in the Brooks model has a mass of approximately $8.37 \mathrm{~g}$ and is comparable to the ICNIRP safety standard of $2 \mathrm{~W} / \mathrm{Kg}$ averaged over $10 \mathrm{~g}$ [17]. The Brooks head is not symmetric as demonstrated by the left eye having a different average SAR from the right one, see Fig. 2. In this research the right eye (as seen from the front) has been arbitrarily chosen to used the symmetric model. There is little change in the average SAR in this eye when the whole head is modelled as against that when the symmetric half head is modelled.

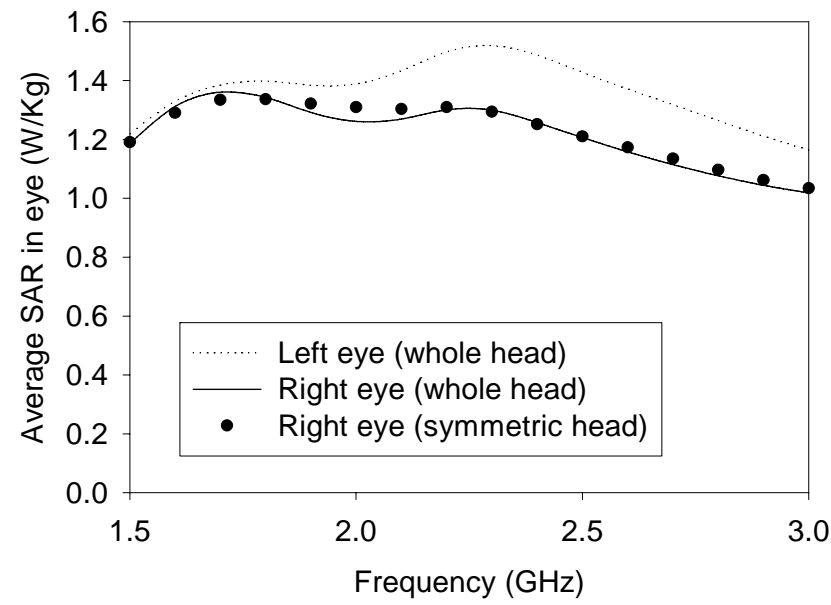

Fig. 2. The average SAR in the eye with and without symmetry.

\section{B. Maximum SAR in the Eye Averaged Over $1 \mathrm{~g}$}

The maximum SAR $1 \mathrm{G}$, is comparable with the ANSI/IEEE standards of $1.6 \mathrm{~W} / \mathrm{Kg}$ [18]. Results for the eye are shown in Fig. 3. Again there is a difference in SAR between the two eyes, but the effect of symmetry has negligible effect on the right eye when using this smaller averaging volume

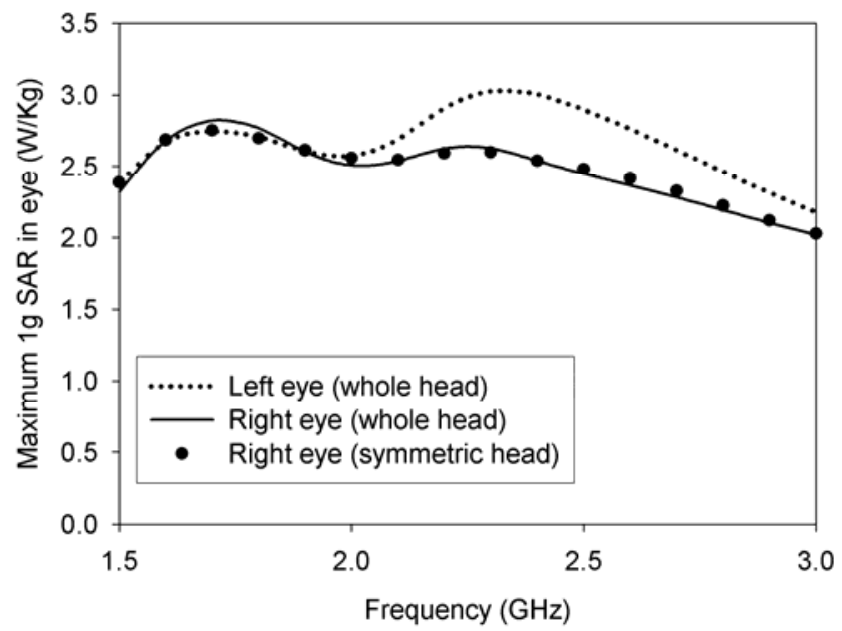

Fig. 3. The maximum 1g SAR in the eye with and without symmetry.

C. Maximum SAR in an individual Yee cell in the eye 
The maximum SAR in an individual Yee cell in the eye is shown in Fig. 4. Reasonable agreement is found between the whole and symmetric heads. The SAR in one cell is extremely sensitive and is thus only an indicative measure of the SAR in the head. Fig. 4 shows the effect of symmetry is only an approximation, which becomes more accurate as a larger volume of the eye is considered.

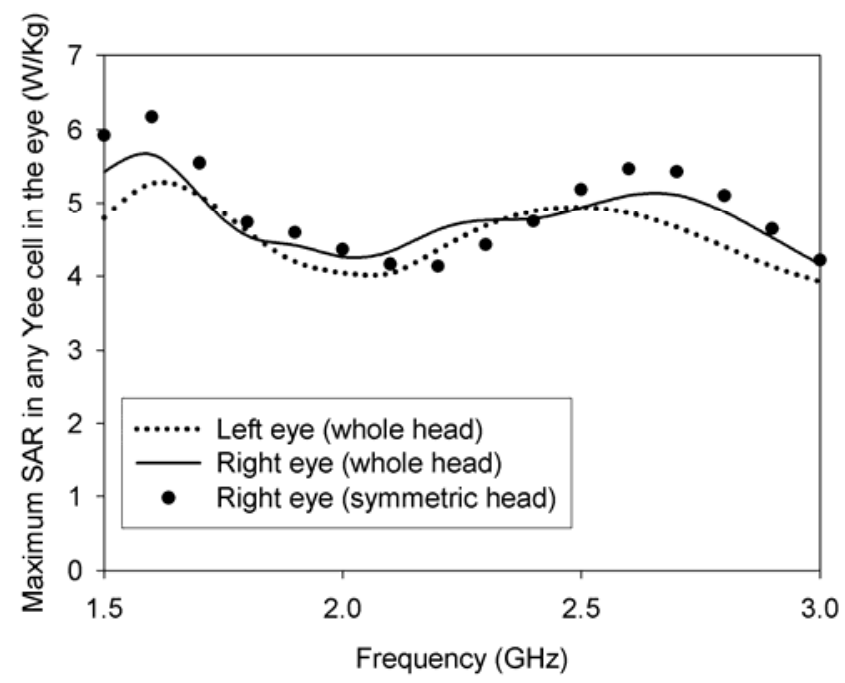

Fig. 4. The maximum SAR in a single cell in the eye with and without symmetry.

D. Maximum $1 g$ SAR in the head

The maximum 1g SAR in the head is shown in Fig. 5.

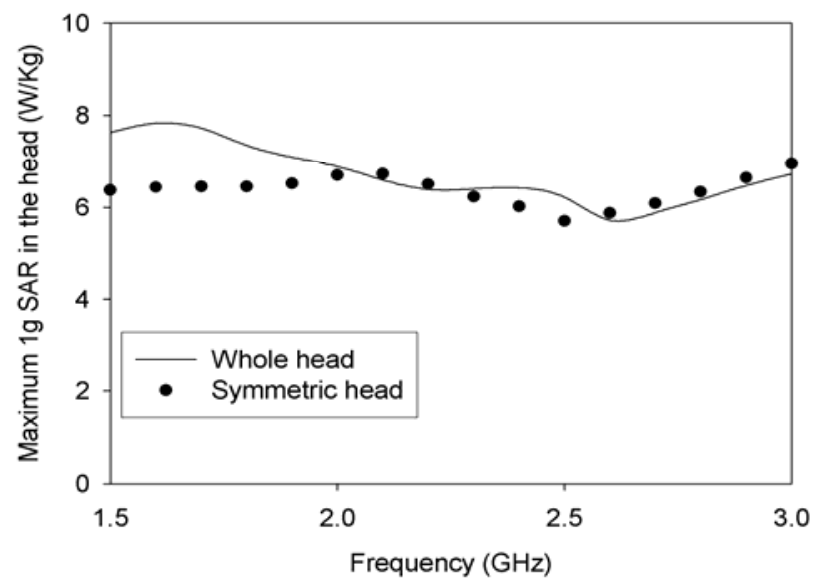

Fig. 5. The maximum $1 \mathrm{~g}$ SAR in the head with and without symmetry.

There is reasonable agreement of this metric with the whole and symmetric heads. The effect of symmetry becomes small above $2 \mathrm{GHz}$. The maximum SAR exists in the nose near the plane of symmetry, thus the averaging volume of the whole head may contain different tissues compared to the symmetric head.

\section{E. Maximum $10 \mathrm{~g}$ SAR in the head}

The maximum $10 \mathrm{~g}$ SAR in the head is shown in Fig. 6. Results are similar to the 1g SAR values in Fig. 5. The agreement is generally reasonable above $2 \mathrm{GHz}$.

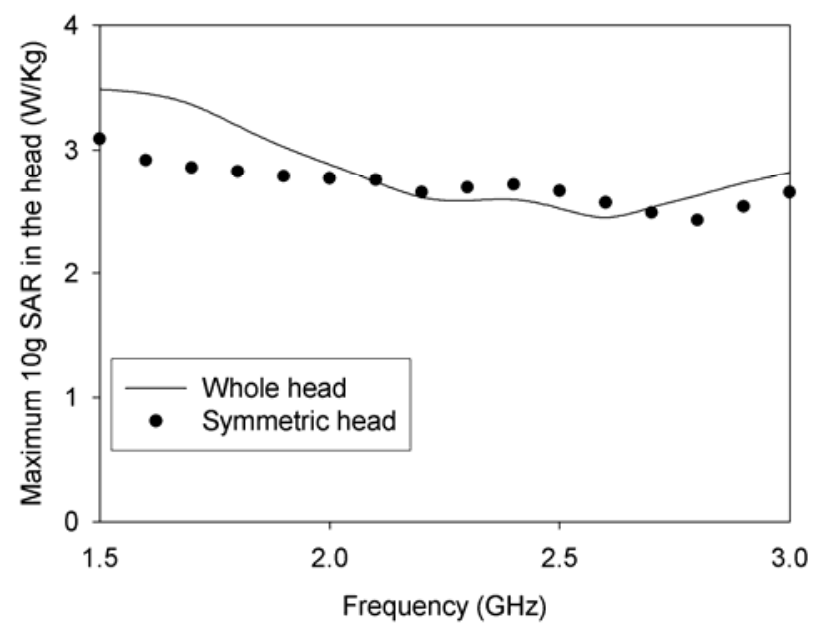

Fig. 6. The maximum $10 \mathrm{~g}$ SAR in the head with and without symmetry.

\section{CONCLUSIONS}

The assumption of using a vertical plane of symmetry in the head excited from the front allows reductions in the computational runtime and memory requirements. Applying symmetry causes negligible loss in accuracy of the SAR in $1 \mathrm{~g}$ of the eye and SAR averaged over the whole eye. The maximum $1 \mathrm{~g}$ and $10 \mathrm{~g}$ SAR in a symmetric head provide reasonable results above $2 \mathrm{GHz}$. Below this frequency the approximation of symmetry becomes less accurate. When the excitation is from the front the maximum SAR values are often located on the central symmetry plane. Therefore the mirrored symmetric head may contain different tissues from the whole head. Also the exterior shape of the nose may not be symmetrical. 


\section{REFERENCES}

1. Tinniswood, A.D., C.M. Furse, and O.P. Gandhi, Computations of SAR distributions for two anatomically based models of the human head using CAD files of commercial telephones and the parallelized FDTD code. IEEE Transactions on Antennas and Propagation, 1998. 46(6): p. 829-833.

2. Gandhi, O., G. Lazzi, and C.M. Furse, Electromagnetic absorption in the human head and neck for mobile telephones at 835 and 1900MHz. IEEE Trans. Microwave Theory Technology, 1996. 44(10): p. 1884-1897.

3. Dimbylow, P.J. and S.M. Mann, Sar Calculations in an Anatomically Realistic Model of the Head for Mobile Communication Transceivers at 900-Mhz and 1.8-Ghz. Physics in Medicine and Biology, 1994. 39(10): p. 15371553.

4. Wainwright, P., Thermal effects of radiation from cellular telephones. Physics in Medicine and Biology, 2000. 45: p. 2363-2372.

5. Martens, L., J. Demoerloose, D. Dezutter, J. Depoorter, and C. Dewagter, Calculation of the Electromagnetic-Fields Induced in the Head of an Operator of a Cordless Telephone. Radio Science, 1995. 30(1): p. 283-290.

6. Dimbylow, P.J. and O.P. Gandhi, Finitedifference time-domain calculations of SAR in a realistic heterogeneous model of the head for plane-wave exposure from $600 \mathrm{MHz}$ to $3 \mathrm{GHz}$. Physics in Medicine and Biology, 1991. 36(8): p. 1075-1089.

7. Hirata, A., S. Matsuyama, and T. Shiozawa, Temperature rises in the human eye exposed to EM waves in the frequency range 0.6-6 GHz. IEEE Transactions on Electromagnetic Compatibility, 2000. 42(4): p. 386-393.

8. Bernardi, P., M. Cavagnaro, S. Pisa, and E. Piuzzi, SAR distribution and temperature increase in an anatomical model of the human eye exposed to the field radiated by the user antenna in a wireless LAN. IEEE Transactions on Microwave Theory and Techniques, 1998. 46(12): p. 2074-2082.

9. Taflove, A., Computational Electrodynamics. The Finite-Difference Time-Domain Method. 1995.

10. Berenger, J.P., A Perfectly Matched Layer for the Absorpton of Electromagnetic Waves. Journal of Computational Physics, 1994. 114: p. 185-200.
11. Brooks-Airforce.

ftp://starview.brooks.af.mil/EMF/dosimetry_mod els/.

12. Hirata, A., H. Watanabe, and T. Shiozawa, SAR and temperature increase in the human eye induced by obliqely incident plane waves. IEEE Trans. Electromagnetic Compatibility, 2002. 44(4): p. 592-594.

13. Gabriel, S., R.W. Lau, and C. Gabriel, The dielectric properties of biological materials: 2 . Measurements in the frequency range $10 \mathrm{~Hz}$ to 20GHz. Physics in Medicine and Biology, 1995. 41: p. 2251-2269.

14. Mason, P.A., W.D. Hurt, T.J. Walters, J.A. D'Andrea, P. Gajsek, K.L. Ryan, D.A. Nelson, K.I. Smith, and J.M. Ziriax, Effects of frequency, permitivity and voxel size on predicted specific absorption rate values in biological tissue during electromagnetic-field exposure. IEEE Trans. Microwave Theory Technology, 2000. 48(11): p. 2050-2058.

15. Caputa, K., M. Okoniewski, and M.A. Stuchly, An algorithm for computations of the power deposition in human tissue. IEEE Antennas and Propagation Magazine, 1999. 41(4): p. 102-107.

16. Whittow, W.G. and R.M. Edwards. A study of changes to specific absorption rates in the human eye close to perfectly conducting spectacles within the radio frequency range 1.5 to 3.0GHz. in Twelfth International Conference on Antennas \& Propagation,. 2003. Exeter, UK.

17. ICNIRP, Guidelines for limiting exposure to time-varying electric, magnetic and electromagnetic fields (up to $300 \mathrm{GHz}$ ). Health Phys, 1998. 74: p. p. 494-522.

18. C95.1-1992, A.I.S., IEEE standard for safety levels with respect to human exposure to radio frequency fields 3kHz to 300GHz. 1992.

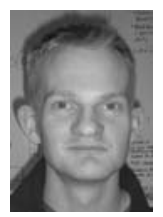

Will G. Whittow was born in Haroldwood, U.K. in 1977. He received the B.Sc in physics from The University of Sheffield, Sheffield, U.K in 2000 and is currently working towards the $\mathrm{PhD}$ degree at The University of Sheffield, Sheffield, U.K.

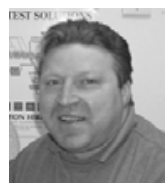

Dr Rob M. Edwards read in Electronic Engineering with Communications at the University of Sheffield, England and studied for his $\mathrm{PhD}$ with the Department's Communications and Radar Group. He is the Director of the Centre for Mobile Communications Research (C4MCR). 